\title{
Radiative boundary-layer flow of an MHD Maxwell fluid with non-linear chemical reaction and heat source in a permeable channel
}

\author{
Amit Parmar*, Shalini Jain
}

Department of Mathematics, University of Rajasthan, Jaipur302004, Rajasthan, India

Corresponding Author Email: amit.198631@gmail.com

https://doi.org/10.18280/ijht.360438

Received: 2 February 2018

Accepted: 30 November 2018

\section{Keywords:}

radiative boundary-layer flow, MHD

Maxwell fluid, non-linear chemical

reaction, porous medium

\begin{abstract}
In this study, we have investigated the radiative boundary-layer flow for MHD Maxwell fluid embedded in a permeable channel. We have considered the various physically effect on fluid flow problem such as non-linear chemical reaction, heat source, thermal radiation, porous medium and thermophoretic effects. The nonlinear PDEs are converted into ODEs. The nondimensional ODEs equations are solved numerically using the bvp4c solver. The impacts of the pertinent parameters such as Re, De, Kn, Q, R, $\tau_{1}$ and $\mathrm{M}$ are depicted through graphically in suction/injection cases. Increasing magnetic field parameter causes rise in thermal boundary layer profiles and exactly reverse effect have been observed for the concentration profile.
\end{abstract}

\section{INTRODUCTION}

The fluid flows in permeable wall channels has play a vital role in several areas such as medical and engineering. Hayat et al. [1-2] investigated Maxwell fluid with radiative MHD in a leaky channel. Vijayalakshmi et al. [3] examined unsteady Casson fluid flow through a vertical channel. Ojjela et al. [4] studied chemically reactive flow of micropolar fluid transfer with ion slip.

Second-grade fluid modal is describing the simplest way of rheological equation exhibited by some non-Newtonian fluids. However, the second-grade fluid does not give suitable results for high Deborah number polymer melts fluids flow. For such situations, the Maxwell model is the best fluid modal in this area. Ibrahim [5] investigated Maxwell fluid flow on stretching sheet with induced magnetic field. Gireesh et al. [6] analyzed Maxwell fluid flow with fluid-particle suspension. Sandeep et al. [7] examined different non-Newtonian nano-particle fluids flow over a stretching surface. Sui et al. [8] proposed Cattaneoe Christov heat flux in Maxwell fluid on a stretching surface. Andersson et al. [9] investigated chemically reactive flow on stretching surface. Prasad et al. [10] examined variable thermos-physical properties of UCM fluid. Mukhopadhyay et al. [11] analyzed UCM fluid flow with chemical reaction. Palani et al. [12] examined UCM fluid flow with non-linear chemical reaction. Naramgari et al. [13] proposed nanofluid flow on porous stretching/shrinking sheet. Parmar et al. [1416] studied the various MHD non-Newtonian fluid flow on different surface under the various physical parameter effect. Jain et al. [17-18] examined MHD flow for viscous fluid due to different surface. Gorla et al. [19-20] proposed convective and chemical reactive slip flow of Williamson fluid over stretching/shrinking sheet with nonlinear thermal radiation.

In this study, we have examined Maxwell fluid flow in a porous channel embedded porous medium. We have considered thermal radiation, inclined MHD, non-linear chemical reaction, heat source and thermophoretic effect on velocity, heat and mass profiles. The effects of physical parameters on the velocity, heat and mass profiles are analyzed with the help of graphs.

$$
y=H / 2
$$

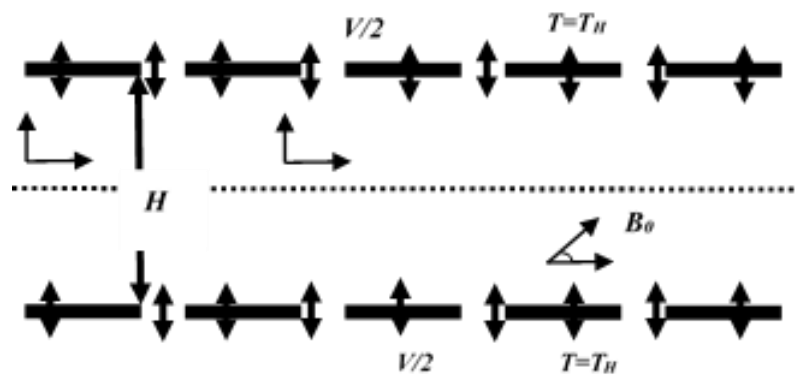

$y=-H / 2$

Figure 1. Physically representation of problem

\section{MATHEMATICAL FORMULATION}

Considered an incomparable radiative boundary-layer flow of non-Newtonian Maxwell fluid with non-linear chemical reaction, heat source embedded porous medium in a channel with permeable walls at $y= \pm \frac{H}{2}$. A magnetic field is applied at an angle $\alpha$ to the fluid flow. On the flow field, no applied voltage or polarization voltage is imposed, therefore electric field has been taken $\vec{E}=0$. Joule heating and hall effect are neglected. Hence the Lorentz force depends only on magnetic field. Upper and lower wall temperature is $T_{H}$. 
by

The continuity, velocity, heat and mass equations are given

$$
\frac{\partial u}{\partial x}+\frac{\partial v}{\partial y}=0
$$

$$
\begin{aligned}
& u \frac{\partial u}{\partial x}+v \frac{\partial u}{\partial y}=v \frac{\partial^{2} u}{\partial y^{2}}-k_{0}\left(u^{2} \frac{\partial^{2} u}{\partial x^{2}}+v^{2} \frac{\partial^{2} u}{\partial y^{2}}+2 u v \frac{\partial^{2} u}{\partial x \partial y}\right) \\
& -\frac{\sigma B_{0}^{2} \sin ^{2} \alpha}{\rho}\left(u+k_{0} v \frac{\partial u}{\partial y}\right)-\frac{v \phi}{k_{p}} u
\end{aligned}
$$

$u \frac{\partial T}{\partial x}+v \frac{\partial T}{\partial y}=\frac{k}{\rho C_{p}} \frac{\partial^{2} T}{\partial y^{2}}-\frac{1}{\rho C_{p}} \frac{\partial q_{r}}{\partial y}+\frac{Q_{0} T}{\rho C_{p}}+\mu\left(\frac{\partial u}{\partial y}\right)^{2}$

$u \frac{\partial C}{\partial x}+v \frac{\partial C}{\partial y}=D_{m} \frac{\partial^{2} C}{\partial y^{2}}-k_{n}(C)^{n}-\frac{\partial}{\partial y}\left(V_{T} C\right)$

where $u(x, y)$ and $\mathrm{v}(\mathrm{x}, \mathrm{y})$ are the horizontal and vertical velocity components, $\rho, v$ and $\rho C_{p}$ are respectively is fluid density, kinematic viscosity and heat capacities of particles.

$\mathrm{T}$ : temperature fluid temperature.

Boundary conditions are following

$$
\begin{aligned}
& v=0, \frac{\partial T}{\partial y}=0, \frac{\partial C}{\partial y}=0 \quad \text { at } y=0 \\
& u \rightarrow 0, v=\frac{V}{2}, T \rightarrow T_{H}, C \rightarrow C_{H} \text { at } y \rightarrow \frac{H}{2}
\end{aligned}
$$

In which $\mathrm{V}>0$ : suction and $\mathrm{V}<0$ : blowing. Following Rosseland approximation $q_{r}$, the radiation heat flux is given

$$
\begin{aligned}
& T^{4} \approx-3 T_{H}{ }^{4}+4 T_{H}{ }^{3} T \\
& \frac{\partial q_{r}}{\partial y}=\frac{\partial}{\partial y}\left(\frac{-4 \sigma}{3 k^{*}} \frac{\partial T^{4}}{\partial y}\right)=\frac{\partial}{\partial y}\left(\frac{-4 \sigma}{3 k^{*}} \frac{\partial\left(-3 T_{H}{ }^{4}+4 T_{H}{ }^{3} T\right)}{\partial r}\right) \\
& =\frac{-16 \sigma T_{H}{ }^{3}}{3 k^{*}} \frac{\partial^{2} T}{\partial y^{2}}
\end{aligned}
$$

Thermophoretic velocity $V_{T}$ is defined is as follows

$$
V_{T}=-\frac{k_{V} v}{T_{r}} \frac{\partial T}{\partial y}
$$

Here $T_{r}$ is the reference temperature, $k_{V}$ is the thermophoretic coefficient [21-22] is given by

$k_{V}=\frac{2 c_{b}\left(\lambda_{g} / \lambda_{p}+c_{t} k_{n}\right)\left(c_{1}+c_{2} e^{-c_{3} / k_{n}}\right)}{\left(1+3 c_{m} k_{n}\right)\left(1+2 \lambda_{g} / \lambda_{p}+2 c_{t} k_{n}\right)}$

Also, where $c_{1}, c_{2}, c_{3}, c_{m}, c_{b}, c_{t}$ are constants and $\lambda_{g}, \lambda_{p}$ are thermal conductivity of the fluid and diffused particles correspondingly and $k_{n}$ is the Knudsen number.

\section{SOLUTION}

We now introduce the following relations for $u, v$ as

$$
\begin{aligned}
& u=-V x^{*} f^{\prime}\left(\mathrm{y}^{*}\right), v=V f\left(\mathrm{y}^{*}\right), x^{*}=\frac{x}{H}, y^{*}=\frac{y}{H}, \\
& \theta\left(\mathrm{y}^{*}\right)=\frac{T}{T_{H}}, \phi\left(\mathrm{y}^{*}\right)=\frac{T}{T_{H}}
\end{aligned}
$$

Equation (2) and (5) thus reduces to the following nondimensional form

$$
\begin{aligned}
& f^{\prime \prime \prime}\left(1-\operatorname{De} f^{2}\right)+2 D e f f^{\prime} f^{\prime \prime}-M \sin ^{2} \alpha\left(\operatorname{Re} f^{\prime}+\operatorname{De} f f^{\prime \prime}\right) \\
& -K_{p} f^{\prime}+\operatorname{Re}\left(f^{\prime 2}-f^{\prime \prime} f\right)=0 \\
& \theta^{\prime \prime}\left(1+\frac{4}{3} R\right)+\operatorname{Pr}\left(E c f^{\prime 2}-\operatorname{Re} f \theta^{\prime}+\mathrm{Q} \theta\right)=0 \\
& \phi^{\prime \prime}+\operatorname{Sc}\left(K_{n} \phi^{n}+\operatorname{Re} f \phi^{\prime}+\tau_{1}\left(\phi \theta^{\prime \prime}+\phi^{\prime} \theta^{\prime}\right)\right)=0
\end{aligned}
$$

Boundary conditions (5) reduces as:

$$
\begin{aligned}
& y=0: \quad f=0, \theta^{\prime}=0, \phi^{\prime}=0 \\
& y=1 / 2: \quad f=1 / 2, f^{\prime}=0, \theta=1, \phi=1
\end{aligned}
$$

Where $\operatorname{Pr}=\frac{\mu C_{p}}{k}:$ Prandtl number, $R=\frac{4 \sigma T_{H}^{3}}{k k^{*}}:$ radiation parameter, $k *$ : thermal radiation parameter, $E c=\frac{V^{2} x^{*^{2}}}{T_{H} C_{p}}$ : Eckert number, $M=\frac{\sigma B_{0}^{2} H}{v \rho}$ : magnetic field parameter, $\lambda$ : the relaxation time, $k_{p}$ : permeability of the porous medium, $Q=$ $\frac{H^{2}}{\mu C_{p}} Q^{*}$ : heat source parameter,: $\tau_{1}=-\frac{k_{v} T_{H}}{T_{r}}$ : thermophoretic parameter, $S c=\frac{v}{D_{m}}$ : Schmidt number, $K_{n}=\frac{H^{2} k_{n}}{v}\left(C_{H}\right)^{n-1}$ : chemical reaction parameter, $k$ : thermal conductivity, $D e=$ $\frac{\lambda V^{2}}{v}$ : Deborah number, $R e=\frac{H V}{v}$ : Reynolds number $(\operatorname{Re}<0$ injection case and $\operatorname{Re}>0$ : suction case ), $K_{p}=\frac{H^{2}}{k_{p}}$ : porosity parameter.

\section{RESULTS AND DISCUSSION}

In this paper, The fix value of physical parameters are $\mathrm{M}=2, \mathrm{Kp}=0.5, \mathrm{Pr}=2, \mathrm{Ec}=1,{ }_{1}=0.1, \mathrm{Sc}=2, \mathrm{R}=0.5, \mathrm{Q}=0.5$, $\mathrm{Kn}=0.5, n=1, \mathrm{De}=0.5, \mathrm{Re}=8, \alpha=\pi / 4$ and excluding the varied value of particular graph with specific two boundary condition such as permeable and melting surface. Several sets of numerical solutions have been carried out for different combinations of pertinent parameters namely, Reynolds number (Re), Deborah number De, chemical reaction parameter $(\mathrm{Kn})$, heat source $(\mathrm{Q})$, radiation parameter $(\mathrm{R})$, thermophoretic parameter $\left(\tau_{1}\right)$ and Hartman number $(\mathrm{M})$ on $f$, $f^{\prime}, \theta$ and $\phi$ profiles. From figs. (2-4) increasing $M$ causes rise in $f$ and $\theta$ profiles and exactly reverse effect have been observed for the $\phi$ profile. Fig (5) shows that enhancement in M, causes increase in $f^{\prime}$ profile for suction case and $f^{\prime}$ suppress for injection case. Physically, magnetic field has produced a drag-like force called the Lorentz force which acts opposite direction on the flow, causing a flow retardation and enhancement thermal energy and concentration. Figs. (67) shows that rising the Kp causes enhancement in $f$ and $f$ 
profile. Fig (8) depicts that enhancement in Kp, keep rising in $\theta$ for suction case and suppress $\theta$ profile for injection case. Fig (9) increases the Kp, suppress $\phi$ in suction case and rising $\phi$ profile in injection case. From Figs. (10-13) it is observed that for non-Newtonian fluid $f, f^{\prime}$ and $\theta$ profiles increases and $\phi$ profile decreases with the increase in $\alpha$. With increase in $\mathrm{R}, \theta$ profile decreases in suction case and increases in injection case, as shown in figs. (14). Generally, increasing values of $\mathrm{R}$, the mean absorption coefficient decreases, which results in rise to the divergence of radiative heat flux. Hence, the rate of radiative heat transferred to the fluid shoot up, so that the fluid temperature increases. Figs (15-16) shows the $\theta$ profile act against the similarity variable $\eta$ for various values of $Q$ and Ec. We examined from these figs that the increases thermal boundary layer thickness as $\mathrm{Q}$ and Ec increases in both suction and injection cases. With increase in $\operatorname{Pr}, \theta$ profile increases in suction case and decreases in injection case, as shown in figs. (17). Prandtl number is ratio of velocity diffusivity to heat diffusivity. Prandtl number can be used to increase the rate of cooling in conducting flows. From figs (18-19) increases the $\tau_{1}$ and $\mathrm{Kn}$ reduces the $\phi$ profiles in both boundary condition. Kn increases the interfacial mass transfer rate. Kn reduces the local concentration, thus increases its concentration gradient and its flux. From fig (20) increasing the value of Sc, reduces the $\phi$ profiles in suction case and increases in injection case. Physically, it is due to the fact that Sc is the ratio of velocity to concentration diffusivities which means that when Sc increases, mass diffusivity decreases and there is a reduction in concentration. Fig (21) increasing $\mathrm{n}$ whereas enhancement the $\phi$ profiles. Table 1 shows the comparison of the present results with the existed results of Hayat at al. [2].

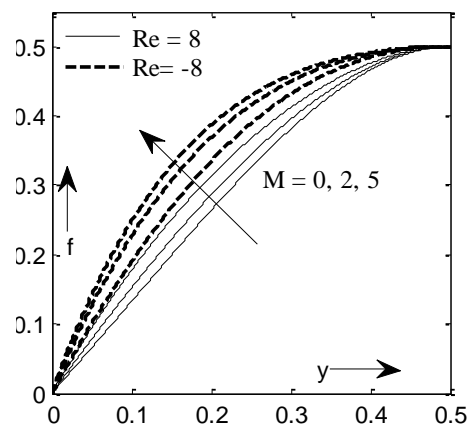

Figure 2. Impact of $\mathrm{M}$ on $f$

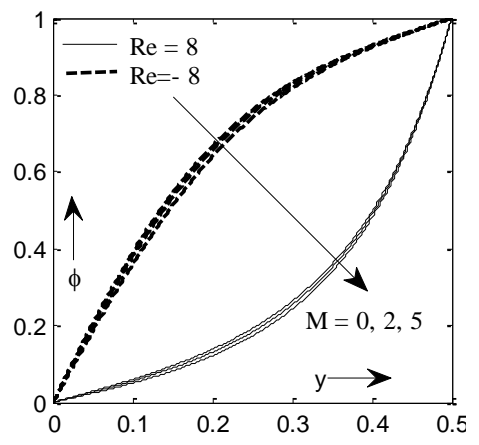

Figure 3. Impact $\mathrm{M}$ on $\phi$ profile

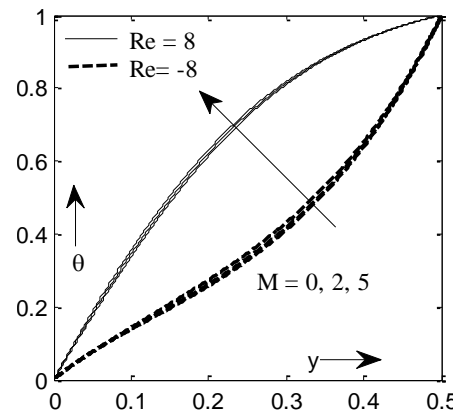

Figure 4. Impact of $M$ on $\theta$

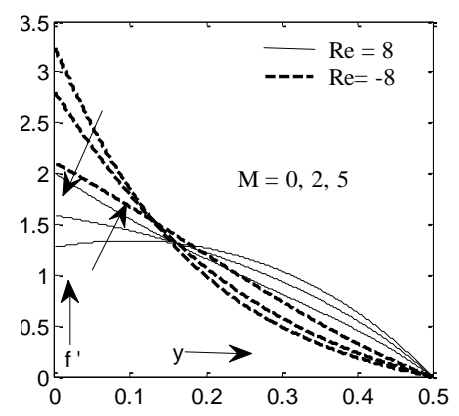

Figure 5. Impact of $\mathrm{M}$ on $f$

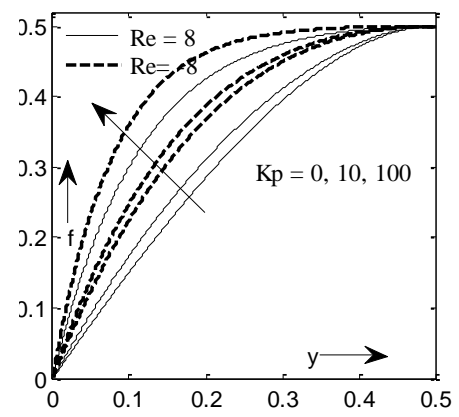

Figure 6. Impact $\mathrm{Kp}$ on $f$ profile

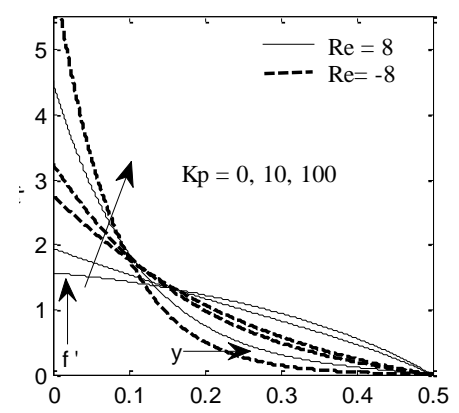

Figure 7. Impact Kp on $f^{\prime}$ profile

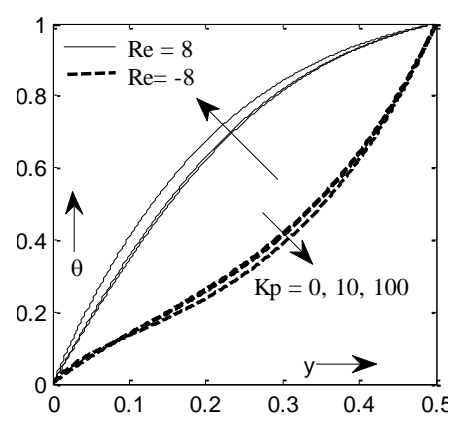

Figure 8. Impact $\mathrm{Kp}$ on $\theta$ profile 


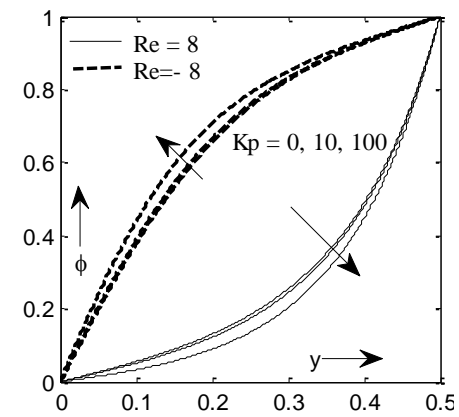

Figure 9. Impact $\mathrm{Kp}$ on $\phi$ profile

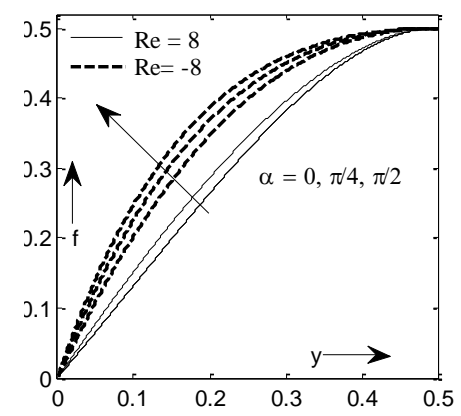

Figure 10. Impact $\alpha$ on $f$ profile

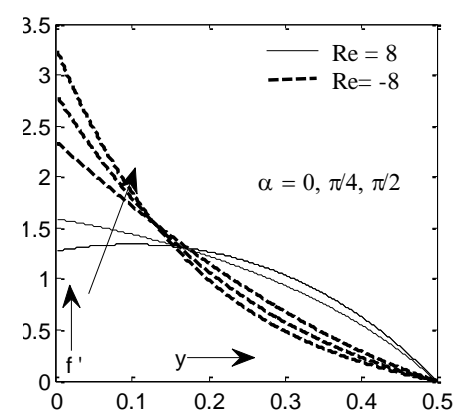

Figure 11. Impact $\alpha$ on $f^{\prime}$ profile

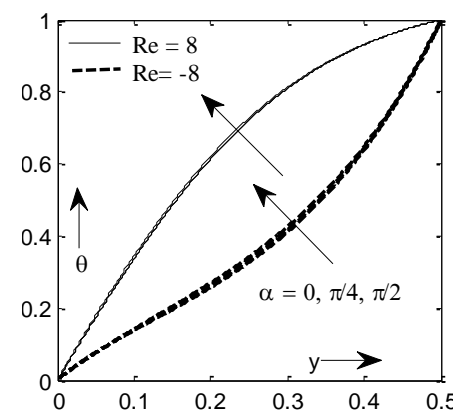

Figure 12. Impact $\alpha$ on $\theta$ profile

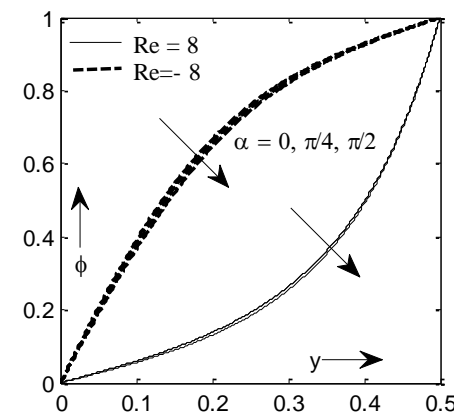

Figure 13. Impact $\alpha$ on $\phi$ profile

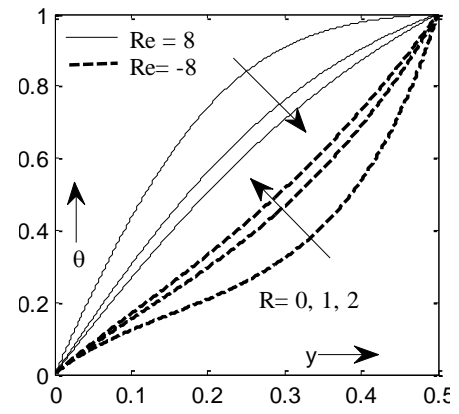

Figure 14. Impact $\mathrm{R}$ on $\theta$ profile

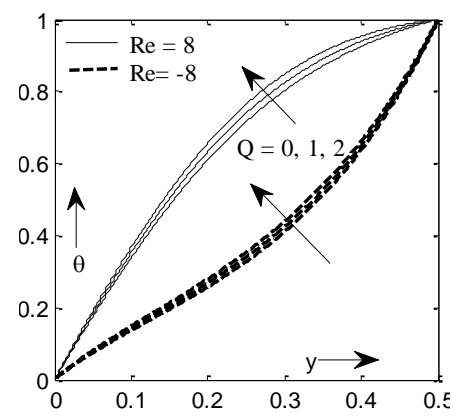

Figure 15. Impact $\mathrm{Q}$ on $\theta$ profile

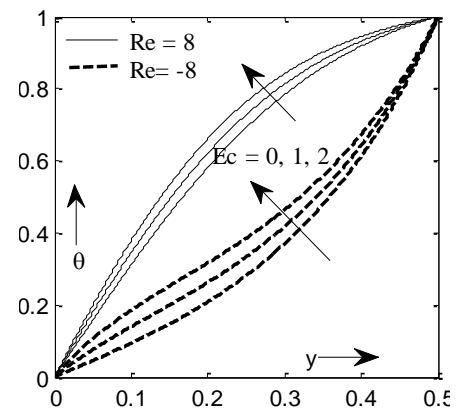

Figure 16. Impact Ec on $\theta$ profile

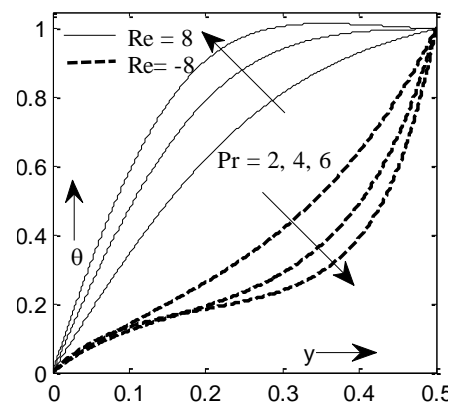

Figure 17. Impact $\operatorname{Pr}$ on $\theta$ profile

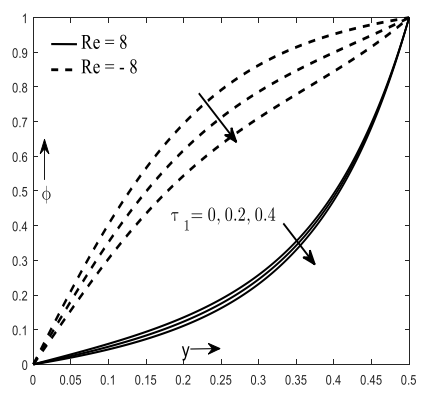

Figure 18. Impact $\tau_{1}$ on $\phi$ profile 


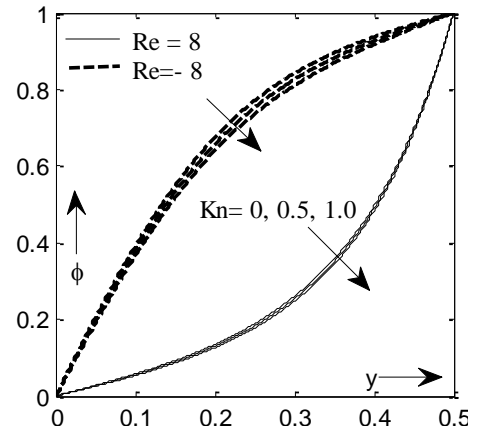

Figure 19. Impact $\mathrm{Kn}$ on $\phi$ profile

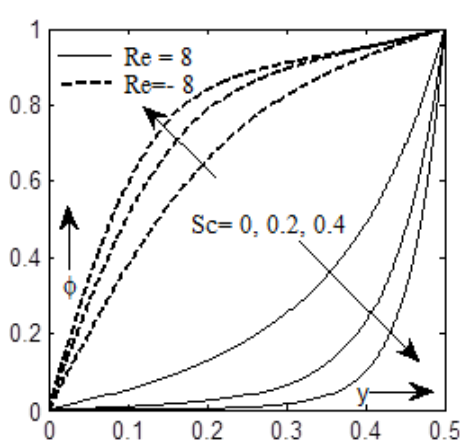

Figure 20. Impact Sc on $\phi$ profile

Table 1. Numerical values of local Nusselt number for the suction case. When $\alpha=\pi / 2$

\begin{tabular}{|c|c|c|c|c|c|c|c|c|}
\hline $\operatorname{Re}$ & $\mathbf{M}$ & Kp & De & $\mathbf{R}$ & Pr & Ec & Hayat at al. [2] & Present study \\
\hline 0 & 1.0 & 1.0 & 1.0 & 0.5 & 0.5 & 0.5 & 1.500753 & 1.5007536 \\
\hline 5 & & & & & & & 1.635831 & 1.63583198 \\
\hline 10 & & & & & & & 1.786391 & 1.78639163 \\
\hline 20 & & & & & & & 2.182340 & 2.18234023 \\
\hline \multirow[t]{24}{*}{5} & 0.0 & & & & & & 1.632181 & 1.63218198 \\
\hline & 2 & & & & & & 1.651119 & 1.65111989 \\
\hline & 4 & & & & & & 1.701858 & 1.70185866 \\
\hline & 8 & & & & & & 1.835777 & 1.83577732 \\
\hline & 1 & 0.0 & & & & & 1.634007 & 1.63400722 \\
\hline & & 4 & & & & & 1.643906 & 1.64390633 \\
\hline & & 8 & & & & & 1.659531 & 1.65953196 \\
\hline & & 16 & & & & & 1.701858 & 1.70185828 \\
\hline & & 1 & 0.0 & & & & 1.632262 & 1.63226296 \\
\hline & & & 1.0 & & & & 1.635831 & 1.63583163 \\
\hline & & & 1.5 & & & & 1.639995 & 1.63999589 \\
\hline & & & 2.0 & & & & 1.647842 & 1.64784212 \\
\hline & & & 1.0 & 0.0 & & & 1.721740 & 1.72174023 \\
\hline & & & & 0.3 & & & 1.659672 & 1.65967234 \\
\hline & & & & 0.6 & & & 1.626706 & 1.62670643 \\
\hline & & & & 0.9 & & & 1.606267 & 1.60626756 \\
\hline & & & & 0.5 & 0.2 & & 0.625678 & 0.62567865 \\
\hline & & & & & 0.4 & & 1.289103 & 1.28910376 \\
\hline & & & & & 0.6 & & 1.993054 & 1.99305428 \\
\hline & & & & & 0.8 & & 2.740532 & 2.74053239 \\
\hline & & & & & 0.5 & 0.5 & 1.635831 & 1.63583151 \\
\hline & & & & & & 1.0 & 3.271662 & 3.27166284 \\
\hline & & & & & & 1.5 & 4.907493 & 4.90749373 \\
\hline & & & & & & 2.0 & 6.543324 & 6.54332462 \\
\hline
\end{tabular}

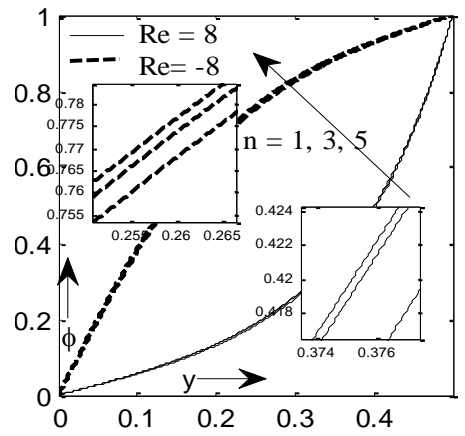

Figure 21. Impact $n$ on $\phi$ profile

\section{CONCLUSION}

Radiative boundary-layer flow of a MHD Maxwell fluid with non-linear chemical reaction, heat source, thermophoretic effect embedded in porous medium is investigated in a permeable channel. The nonlinear PDEs are convert into an ODEs and solved numerically using the bvp4c solver. Our computations have indicated that:

- Increase in M, Kp enhances $f$ profile

- Increase in $\mathrm{M}$ rising $f^{\prime}$ for suction case and $f^{\prime}$ suppress for injection case.

- Increase in Q and Ec enhances the $\theta$ profile.

- Increase in $\tau_{1}$ and $\mathrm{Kn}$ increases the $\phi$ profile.

- Increase in $\operatorname{Pr}$ reduces the $\theta$ profile in injection condition and rising $\theta$ profile in suction condition.

\section{REFERENCES}

[1] Abbas Z, Sajid M, Hayat T. (2006). MHD boundarylayer flow of an upper-convected Maxwell fluid in a porous channel. Theor. Comput. Fluid Dyn 20: 229-238. https://doi.org/10.1007/s00162-006-0025-y

[2] Hayat T, Sajjad R, Abbas Z, Sajid M, Hendi AA. (2011). Radiation effects on MHD flow of Maxwell fluid in a channel with porous medium. International Journal of 
Heat and Mass Transfer 54: 854-862. https://doi.org/10.1016/j.ijheatmasstransfer.2010.09.069

[3] Vijayalakshmi R, Sarojamma G, Sreelakshmi K, Sandhya G. (2017). Unsteady flow of a Casson fluid through a vertical channel with walls of expansion and contraction. IJRISE, SI: 2017.

[4] Ojjela O, Kumar NN. (2016). Chemically reacting micropolar fluid flow and heat transfer between expanding or contracting walls with ion slip, Soret and Dufour effects. Alexandria Engineering Journal 55: 1683-1694.

[5] Ibrahim W. (2016). The effect of induced magnetic field and convective boundary condition on MHD stagnation point flow and heat transfer of upper-convected Maxwell fluid in the presence of nanoparticle past a stretching sheet. Propulsion and Power Research 5(2): 164-175. https://doi.org/10.1109/tnano.2014.2375912

[6] Gireesh BJ, Mahanthesh B, Gorla RSR, Krupalakshmi KL. (2016). Mixed convection two-phase flow of Maxwell fluid under the influence of non-linear thermal radiation, non-uniform heat source/sink and fluidparticle suspension. https://doi.org/10.1016/j.asej.2016.02.008

[7] Sandeep NO, Sulochan C. (2016). Momentum and heat transfer behaviour of Jeffrey, Maxwell and Oldroyd-B nanofluids past a stretching surface with non-uniform heat source/sink. https://doi.org/10.1016/j.asej.2016.02.008

[8] Sui J, Zheng L, Zhang X. (2016). Boundary layer heat and mass transfer with Cattaneoe Christov doublediffusion in upper-convected Maxwell Nanofluid past a stretching sheet with slip velocity. International Journal of Thermal Sciences 104: 461-468.

[9] Andersson HI, Hansen OR, Holmedal B. (1994). Diffusion of a chemically reactive species from a stretching sheet. Int J Heat Mass Transfer 37: 659-64. https://doi.org/10.1016/0017-9310(94)90137-6

[10] Prasad KV, Sujatha A, Vajravelu K, Pop I. (2012). MHD flow and heat transfer of a UCM fluid over a stretching surface with variable thermos-physical properties. Meccanica 47: 1425-39. https://doi.org/10.1007/s11012-011-9526-X

[11] Mukhopadhyay S, Golam AM., Wazed AP. (2013). Effects of transpiration on unsteady MHD flow of an UCM fluid passing through a stretching surface in the presence of a first order chemical reaction. Chin Phys B 22: $\quad 124701 . \quad$ https://doi.org/10.1088/1674$1056 / 22 / 12 / 124701$

[12] Palani S, Kumar BR, Kameswaran PK. (2016). Unsteady MHD flow of an UCM fluid over a stretching surface with higher order chemical reaction. Ain Shams $\begin{array}{llll}\text { Engineering } & \mathrm{J} & 7 \text { 399-408 }\end{array}$ https://doi.org/10.1016/j.asej.2015.11.021

[13] Naramgari S, Sulochana C. (2016). MHD flow over a permeable stretching/shrinking sheet of a nanofluid with suction/injection. Alexandria Engineering Journal 55: 819-827. https://doi.org/10.1016/j.aej.2016.02.001

[14] Parmar A. (2017). MHD Falkner-Skan flow of Casson fluid and heat transfer with variable property past a moving wedge. International Journal of Applied and Computational https://doi.org/10.1007/s40819-017-0373-x

[15] Parmar A. (2017). Unsteady convective boundary layer

flow for MHD Williamson fluid over an inclined porous stretching sheet with non-linear radiation and heat source. International Journal of Applied and Computational https://doi.org/10.1007/s40819-017-0373-4

[16] Parmar A, Jain S. (2017). Comparative study of flow and heat transfer behavior of Newtonian and nonNewtonian fluids over a permeable stretching surface Global and stochastic analysis. SI: 41-50.

[17] Jain S, Choudhary R. (2017). Soret and Dufour effects on MHD fluid flow due to moving permeable cylinder with radiation. Global and Stochastic Analysis. SI: 7584.

[18] Jain S, Choudhary R. (2015). Effects of MHD on boundary layer flow in porous medium due to exponentially shrinking sheet with slip. Procedia Engineering 127: 1203-1210. https://doi.org/10.1016/j.proeng.2015.11.464

[19] Gorla RSR, Gireesh BJ. (2016). Dual solutions for stagnation-point flow and convective heat transfer of a Williamson nanofluid past a stretching/shrinking sheet. Heat Mass Transfer 52: 1153-1162. https://doi.org/10.1007/s00231-015-1627-y

[20] Gorla RSR, Prasanna KBC, Gireesh BJ, Krishnamurthy MR. (2016). Effects of chemical reaction and nonlinear thermal radiation on Williamson nanofluid slip flow over a stretching sheet embedded in a porous medium. J. Aerosp. Eng 29(5): 04016019.

[21] Rashad AM. (2008). Influence of radiation on MHD free convection from a vertical flat plate embedded in porous media with thermophoretic deposition of particles. Communications in Nonlinear Science and Numerical Simulation 13: 2213-2222. https://doi.org/10.1016/j.cnsns.2007.07.002

[22] Noor NFM, Abbasbandy S, Hashim I. (2012). Heat and mass transfer thermophoretic MHD flow over an inclined radiate isothermal permeable surface in the presence of heat source/sink. Int. $\mathrm{J}$ of Heat and Mass Transfer 55: 2122-2128. https://doi.org/0.1016/j.ijheatmasstransfer.2011.12.015

\section{NOMENCLATURE}

$\mathrm{R}$

$\mathrm{V}$

$\mathrm{Re}$

De

$\mathrm{k}$

$\operatorname{Pr}$

Q

$\mathrm{M}$

$\mathrm{Sc}$

$\mathrm{Kn}$

$\mathrm{n}$

\section{Greek symbols}

$\theta$

$\phi$

$\alpha$

$\rho$

$\alpha$

$\rho$

radiation parameter

Suction /injection parameter

Reynold number

Deborah number

thermal conductivity

Prandtl number

Heat source

Magnetic field parameter

Schmit number

Chemical reaction

Order of Chemical reaction
Dimensionless temperature

Dimensionless concentration. Inclined angle of magnetic field Fluid density 